LPTENS-05/13

\title{
Dual Form of the Paperclip Model
}

\author{
Sergei L. Lukyanov ${ }^{1,2}$ and Alexander B. Zamolodchikov ${ }^{1,2,3}$ \\ ${ }^{1}$ NHETC, Department of Physics and Astronomy \\ Rutgers University \\ Piscataway, NJ 08855-0849, USA \\ ${ }^{2}$ L.D. Landau Institute for Theoretical Physics \\ Chernogolovka, 142432, Russia \\ and \\ ${ }^{3}$ Chaire Internationale de Recherche Blaise Pascal \\ Laboratoire de Physique Théorique de l'Ecole Normale Supérieure \\ 24 rue Lhomond, Paris Cedex 05, France
}

\begin{abstract}
The "paperclip model" is 2D model of Quantum Field Theory with boundary interaction defined through a special constraint imposed on the boundary values of massless bosonic fields (hep-th/0312168). Here we argue that this model admits equivalent "dual" description, where the boundary constraint is replaced by special interaction of the boundary values of the bosonic fields with an additional boundary degree of freedom. The dual form involves the topological $\theta$-angle in explicit way.
\end{abstract}

October 2005 


\section{Introduction}

In this work we describe the dual form of the "paperclip model" of boundary interaction in 2D Quantum Field Theory. The paperclip model was introduced in Ref. [1], where its basic properties are discussed. The model involves two-component Bose field $\mathbf{X}(\sigma, \tau)=(X(\sigma, \tau), Y(\sigma, \tau))$ which lives on a semi-infinite cylinder with Cartesian coordinates $(\sigma, \tau), \sigma \geq 0, \tau \equiv$ $\tau+1 / T^{1}$. In the bulk, i.e. at $\sigma>0$, the dynamics is described by the free-field action:

$$
\mathscr{A}_{\text {bulk }}[X, Y]=\frac{1}{4 \pi} \int_{0}^{1 / T} \mathrm{~d} \tau \int_{0}^{\infty} \mathrm{d} \sigma\left[\left(\partial_{\nu} X\right)^{2}+\left(\partial_{\nu} Y\right)^{2}\right]
$$

The interaction takes place at the boundary at $\sigma=0$, due to a non-linear boundary constraint: the boundary values $\mathbf{X}_{B}=\left.\left(X_{B}, Y_{B}\right) \equiv \mathbf{X}\right|_{\sigma=0}$ of the field $\mathbf{X}$ are restricted to the "paperclip curve"

$$
r \cosh \left(\frac{X_{B}}{2 b}\right)-\cos \left(\frac{Y_{B}}{2 a}\right)=0, \quad\left|Y_{B}\right| \leq \pi a,
$$

Here $a, b$, and $r$ are real positive parameters, the first two being related as follows ${ }^{2}$,

$$
a^{2}-b^{2}=\frac{1}{2}
$$

As usual, the non-linear constraint requires renormalization, but one can check (up to two loops) that the RG transformation affects the curve (2) only through renormalization of the parameter $r$, which "flows" according to the equation

$$
\frac{E_{*}}{E}=4 b^{2}\left(1-r^{2}\right) r^{4 b^{2}},
$$

where $E$ is the RG energy, and $E_{*}$ is the integration constant of the RG equation, which sets up the "physical scale" in the model. As in [1], in what follows we always take the scale $E$ proportional to the temperature $T$, namely

$$
E=2 \pi T .
$$

\footnotetext{
${ }^{1}$ Euclidean formulation of the theory is implied. Due to the compactification $\tau \equiv$ $\tau+1 / T$ it is equivalent to the Matsubara representation of the $1+1$ dimensional theory at thermodynamic equilibrium at the temperature $T$.

${ }^{2}$ The parameter $n$ used in [1] is related to $a$ and $b$ as

$$
a=\frac{\sqrt{n+2}}{2}, \quad b=\frac{\sqrt{n}}{2} .
$$
}


It is useful to introduce also the external field $\mathbf{h}=\left(h_{x}, h_{y}\right)$ which couples to the boundary values $\mathbf{X}_{B}$, i.e. to add the boundary term

$$
\mathscr{A}_{\mathrm{h}}\left[X_{B}, Y_{B}\right]=\int_{0}^{1 / T} \mathrm{~d} \tau\left(h_{x} X_{B}+h_{y} Y_{B}\right)
$$

to the action (11). Then, the first object of interest is the partition function,

$$
Z_{0}=\int \mathcal{D} X \mathcal{D} Y \mathrm{e}^{-\mathscr{A}_{\text {bulk }}[X, Y]-\mathscr{A}_{\mathrm{h}}\left[X_{B}, Y_{B}\right]},
$$

where the functional integration is over all fields $\mathbf{X}(\sigma, \tau)$ obeying the boundary constraint (2).

General definition of the model involves additional parameter, the topological angle $\theta$. Topologically, the paperclip curve (2) is a circle, hence the configuration space for the field $\mathbf{X}(\sigma, \tau)$ consists of sectors characterized by an integer $w$, the number of times the boundary value $\mathbf{X}_{B}$ winds around the paperclip curve when one goes around the boundary at $\sigma=0$. The contributions from the topological sectors can be weighted with the factors $\mathrm{e}^{\mathrm{i} w \theta}$. Thus, in general

$$
Z_{\theta}=\sum_{w=-\infty}^{\infty} \mathrm{e}^{\mathrm{i} w \theta} Z^{(w)}
$$

where $Z^{(w)}$ is the functional integral (7) taken over the fields from the sector $w$ only. Physics of the model, in particular its infrared (i.e. low temperature) behavior, depends on $\theta$ in a significant way (see $[1,2]$ for details).

The ultraviolet (high- $T$ ) limit of the paperclip model is understood in terms of the conformally invariant "hairpin model" of boundary interaction [1]. In this limit the parameter $r$ tends to zero, and the paperclip curve becomes a composition of two "hairpins", as shown in Fig. 1.

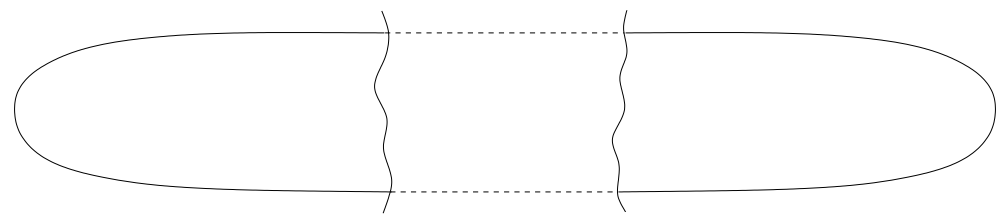

Figure 1: The paperclip formed by junction of two hairpins.

The hairpin model is defined by replacing the paperclip constraint (2) by the non-compact "hairpin" curve $\frac{r}{2} \exp \left( \pm \frac{X_{B}}{2 b}\right)=\cos \left(\frac{Y_{B}}{2 a}\right)$. We refer to 
this model as the "left" or the "right" hairpin, depending on the sign in the exponential; the two models are related by simple field transformation $(X, Y) \rightarrow(-X, Y))$. Note that the left hairpin corresponds to the right part in Fig. 2, and vice versa (just like the way a human brain is wired to the rest of the body). The left (right) hairpin model is conformally invariant with the linear dilaton $D(\mathbf{X})=\frac{X}{2 b}\left(D(\mathbf{X})=-\frac{X}{2 b}\right)$. More details on the hairpin model can be found in [1] and in Section[2 below.

The paperclip model has many features in common with the so-called "sausage" sigma model studied in Ref. [3]. The UV splitting of the paperclip into the hairpins is analogous to the UV splitting of the sausage into two semi-infinite "cigars" (see [3]). Like the sausage sigma model, the paperclip model seems to be integrable at two values of the topological angle, $\theta=0$ and $\theta=\pi$. The sausage model is known to admit a dual description, where the non-trivial metric is replaced by certain potential ("tachion") term in the action [4]. This analogy is one of the reasons to expect that a similar dual representation exists for the paperclip model. The aim of this work is to introduce the dual representations of both the hairpin model and the paperclip model.

In this paper we argue that the paperclip model is equivalent (or "dual") to another model of boundary interaction. The dual model also involves a two-component Bose field $(X(\sigma, \tau), \tilde{Y}(\sigma, \tau)$ ) (where $\tilde{Y}$ is interpreted as the T-dual ${ }^{3}$ of $Y$ ) on the semi-infinite cylinder, which has free-field dynamics in the bulk, and obeys no constraint at the boundary $\sigma=0$; instead it interacts with an additional boundary degree of freedom. It is best to discuss the dual model in terms of its Hamiltonian representation, with the cyclic coordinate $\tau \equiv \tau+1 / T$ taken as the Euclidean (or Matsubara) time. In this picture the partition function (8) admits the dual representation as the trace

$$
Z_{\theta}=\operatorname{Tr}_{\tilde{\mathcal{H}}}\left[\mathrm{e}^{-\frac{\hat{H}}{T}}\right]
$$

taken over the space $\tilde{\mathcal{H}}=\mathcal{H}_{X, \tilde{Y}} \otimes \mathbb{C}^{2}$, where $\mathcal{H}_{X, \tilde{Y}}$ is the space of states of the two-component boson $(X(\sigma), \tilde{Y}(\sigma))$ on the half-line $\sigma \geq 0$ (with no constraint at $\sigma=0$ ) and $\mathbb{C}^{2}$ is the two-dimensional space representing the new boundary degree of freedom. The dual Hamiltonian in (9) consists of the bulk and the boundary parts, $\hat{H}=\hat{H}_{\text {bulk }}+\hat{H}_{\text {boundary }}$. The bulk part is just the free-field Hamiltonian

$$
\hat{H}_{\mathrm{bulk}}=\frac{1}{4 \pi} \int_{0}^{\infty} \mathrm{d} \sigma\left[\Pi_{X}^{2}+\Pi_{\tilde{Y}}^{2}+\left(\partial_{\sigma} X\right)^{2}+\left(\partial_{\sigma} \tilde{Y}\right)^{2}\right]
$$

\footnotetext{
${ }^{3}$ The T-dual of the free massless field is defined as usual, through the relations: $\partial_{\tau} \tilde{Y}=$ i $\partial_{\sigma} Y$ and $\partial_{\sigma} \tilde{Y}=-\mathrm{i} \partial_{\tau} Y$.
} 
where $\left(\Pi_{X}, \Pi_{\tilde{Y}}\right)$ are momenta conjugated to the field operators $(X, \tilde{Y})^{4}$ acting in $\mathcal{H}_{X, \tilde{Y}}\left(\hat{H}_{\text {bulk }}\right.$ acts as identity in the $\mathbb{C}^{2}$ component of $\left.\tilde{\mathcal{H}}\right)$. The boundary term describes coupling of the boundary values $\left.\left(X_{B}, \tilde{Y}_{B}\right) \equiv(X, \tilde{Y})\right|_{\sigma=0}$ of the fields to the additional boundary degree of freedom represented by $\mathbb{C}^{2}\left(\sigma_{ \pm}\right.$and $\sigma_{3}$ are the Pauli matrices acting in $\left.\mathbb{C}^{2}\right)$,

$$
\hat{H}_{\text {boundary }}=h_{x} X_{B}+\pi a h_{y} \sigma_{3}+\hat{V},
$$

where $^{5}$ :

$$
\hat{V}=\mu_{B}\left[\sigma_{+} \cosh \left(b X_{B}-\mathrm{i} \frac{\theta}{2}\right) \mathrm{e}^{\mathrm{i} a \tilde{Y}_{B}}+\sigma_{-} \cosh \left(b X_{B}+\mathrm{i} \frac{\theta}{2}\right) \mathrm{e}^{-\mathrm{i} a \tilde{Y}_{B}}\right],
$$

with $\mu_{B}$ related to the scale $E_{*}$ in (44) as

$$
\mu_{B}=\sqrt{\frac{2 E_{*}}{\pi}} .
$$

In Eqs.(11), (12), $a, b$ and $\left(h_{x}, h_{y}\right)$ are the same as in (21), (16).

\section{Dual form of the hairpin}

In this section we describe the dual form of the conformal hairpin model. To be definite, throughout this section we concentrate attention on the left hairpin model; the right hairpin is obtained by reflection $X \rightarrow-X$. The left hairpin boundary constraint has the form

$$
\frac{r_{*}}{2} \exp \left(\frac{X_{B}}{2 b}\right)=\cos \left(\frac{Y_{B}}{2 a}\right), \quad\left|Y_{B}\right| \leq \pi a .
$$

Here $r_{*}$ relates to the energy scale (15) in a simple way

$$
\frac{E_{*}}{2 \pi T}=4 b^{2}\left(r_{*}\right)^{4 b^{2}} \text {. }
$$

The boundary state of the (left) hairpin model was described in [1],

$$
\left\langle B_{\supset}\right|=\int_{\mathbf{P}} \mathrm{d}^{2} \mathbf{P} B_{\supset}(\mathbf{P})\left\langle I_{\mathbf{P}}\right|,
$$

where $\left\langle I_{\mathbf{P}}\right|$ are the Ishibashi states associated with the $W$-algebra of the hairpin model (see [1] and Section 2.1]below), $\mathbf{P}$ is the zero-mode momentum

\footnotetext{
${ }^{4}$ Normalization is such that, for instance, $\left[X(\sigma), \Pi_{X}\left(\sigma^{\prime}\right)\right]=2 \pi \mathrm{i} \delta\left(\sigma-\sigma^{\prime}\right)$.

${ }^{5}$ We assume canonical conformal normalization of the boundary vertex operators (see e.g. Eq.(48) involved in this interaction term.
} 
of the free boson $\mathbf{X}$, and the amplitude $B_{\supset}(\mathbf{P})$ has the following explicit form in terms of the components $(P, Q)$ of the vector $\mathbf{P}$

$$
B_{\supset}(P, Q)=\mathrm{g}_{D}^{2} r_{*}^{-2 \mathrm{i} b P} \frac{2 b \Gamma(2 \mathrm{i} b P) \Gamma\left(1+\mathrm{i} \frac{P}{2 b}\right)}{\Gamma\left(\frac{1}{2}-a Q+\mathrm{i} b P\right) \Gamma\left(\frac{1}{2}+a Q+\mathrm{i} b P\right)},
$$

where $\mathrm{g}_{D}=2^{-1 / 4}$. The amplitude $B_{\supset}(P, Q)$ coincides with the partition function of the hairpin model on the semi-infinite cylinder of circumference $1 / T$ :

$$
Z_{\supset}\left(h_{x}, h_{y}\right)=B_{\supset}(P, Q),
$$

where the dependence of $Z_{\supset}$ on the parameters $h_{x}, h_{y}$ is brought about through the linear boundary term (6), and $P, Q$ in the right hand side are related to the these parameters as follows

$$
P=\mathrm{i} \frac{h_{x}}{T}, \quad Q=\mathrm{i} \frac{h_{y}}{T} .
$$

\section{$2.1 W$-algebra and dual potential}

As was explained in [1], the hairpin boundary condition is conformally invariant (with linear dilaton $X / b$ ), and moreover it has extended conformal symmetry with respect to certain $W$-algebra. Here we use the notation $W_{\supset}$ for the $W$-algebra of the left hairpin model. It is generated by a set of local currents $\left\{W_{s}(z), s=2,3,4 \ldots\right\}$ (built from derivatives of the free fields $X, Y)$ which commute with two "screening operators" 6 ,

$$
\oint_{z} \mathrm{~d} w W_{s}(z) \mathrm{e}^{b X \pm \mathrm{i} a \tilde{Y}}(w, \bar{w})=0 .
$$

Then, it is almost trivial observation that this $W$-symmetry is present in any model of boundary interaction which has no boundary constraint, but instead whose action has an additional boundary potential term

$$
\mathscr{A}_{\mathrm{BP}}=\int_{0}^{1 / T} \mathrm{~d} \tau\left[S_{+} \mathrm{e}^{b X_{B}+\mathrm{i} a \tilde{Y}_{B}}+S_{-} \mathrm{e}^{b X_{B}-\mathrm{i} a \tilde{Y}_{B}}\right] .
$$

Here $S_{ \pm}$may be either c-numbers, or more generally any non-trivial boundary degrees of freedom whose own dynamics is "topologically invariant", i.e.

\footnotetext{
${ }^{6}$ Note that we write the screening operators in terms of the T-dual field $\tilde{Y}$. This is done in preparation to the discussion of the dual hairpin below. At this point the distinction

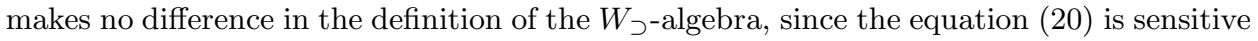
to the holomorphic parts of the fields $X$ and $Y$ only.
} 
invariant with respect to any diffeomorphism $\tau \rightarrow f(\tau)$ of the boundary. Since such boundary interaction has the hairpin $W$-algebra as its symmetry, it seems natural to expect that under appropriate choice of the boundary degree of freedom $S_{ \pm}$the model with the boundary potential (21) is equivalent (or, in modern speak, "dual") to the left hairpin model.

Because of the essentially quantum nature of the boundary degree of freedom $S_{ \pm}$(see below), it will be convenient to discuss in terms of the Hamiltonian representation of the model, as was mentioned in Introduction. The partition function of the left hairpin model is written as the trace $\operatorname{Tr}\left[\mathrm{e}^{-\frac{\hat{H}}{T}}\right]$, where $\hat{H}$ is the sum $\hat{H}_{\text {bulk }}+\hat{V}_{\supset}$ of the bulk free-field Hamiltonian (10), and the boundary term

$$
\hat{V}_{\supset}=\mathbb{S}_{+} \mathrm{e}^{b X_{B}+\mathrm{i} a \tilde{Y}_{B}}+\mathbb{S}_{-} \mathrm{e}^{b X_{B}-\mathrm{i} a \tilde{Y}_{B}},
$$

corresponding to the term (21) in the action. Here $\mathbb{S}_{ \pm}$are the operators associated with the boundary degree of freedom $S_{ \pm}(\tau)$ in (21). The latter operators must commute with the field operators, i.e. $\left[\mathbb{S}_{ \pm}, \mathbf{X}(\sigma)\right]=$ $\left[\mathbb{S}_{ \pm}, \boldsymbol{\Pi}(\sigma)\right]=0$ at any $\sigma$, lest the $W$-symmetry of the theory be violated, but the commutation relations among the boundary observables $\mathbb{S}_{ \pm}$themselves are not fixed a priori. Our goal here is to identify the algebra of these operators in the dual hairpin model, as well as its representation $\rho$.

Some relations can be inferred from the following simple argument. The boundary potential term (21) vanishes in the limit $X \rightarrow-\infty$. In the absence of this term both fields $X$ and $\tilde{Y}$ would obey the von Neumann (free) boundary conditions. Equivalently, in the absence of the potential term the field $Y$ would obey the Dirichlet (fixed) boundary condition, i.e. the boundary values $\left(X_{B}, Y_{B}\right)$ would lay on a straight brane parallel to the $X$-axis. Note that in the same limit $X \rightarrow-\infty$ the right hairpin curve (14) becomes a composition of two parallel branes,

$$
Y_{B} \rightarrow \pm \pi a \text { as } X_{B} \rightarrow-\infty
$$

separated by the distance $2 \pi a$. In the full hairpin curve, these two "legs" are bridged at the right, allowing for a passage from the upper leg to the lower leg and vice versa. In the dual representation of the hairpin model such passages should be attributed to two terms in the boundary potential (21). The boundary vertex operators $\mathrm{e}^{ \pm \mathrm{i} a \tilde{Y}_{B}}(\tau)$ create jumps in the boundary value of $Y$ exactly of the desired magnitude, $Y_{B}(\tau+0)-Y_{B}(\tau-0)= \pm 2 \pi a$. The fact that the hairpin has only two "legs" (23) clearly suggests that the 
operators $\mathbb{S}_{ \pm}$in (22) must obey the "fermionic" relations ${ }^{7}$

$$
\mathbb{S}_{+}^{2}=\mathbb{S}_{-}^{2}=0
$$

So far in the discussion of the dual hairpin we have ignored the coupling (6) to the external field $\left(h_{x}, h_{y}\right)$. Adding the term $h_{x} X_{B}$ to the dual hairpin Hamiltonian is straightforward, but in the description in terms of the Tdual field $\tilde{Y}$ the term $h_{y} Y_{B}$ would be non-local. One can circumvent this difficulty by introducing the "fermion number" operator $\mathbb{N}$, associated with the boundary degrees of freedom $\mathbb{S}_{ \pm}$, which satisfies with them the following commutation relations:

$$
\left[\mathbb{N}, \mathbb{S}_{+}\right]=2 \mathbb{S}_{+}, \quad\left[\mathbb{N}, \mathbb{S}_{-}\right]=-2 \mathbb{S}_{-} .
$$

Then one can check that the sum $Y_{B}-\pi a \mathbb{N}$ commutes with the Hamiltonian (22). Therefore, in the presence of the external field $\mathbf{h}$, expected form of the full Hamiltonian of the dual hairpin model is

$$
\hat{H}=\hat{H}_{0}+\hat{V}_{\supset}
$$

where $\hat{V}_{\supset}$ is the boundary potential operator (22), and

$$
\hat{H}_{0}=\hat{H}_{\mathrm{bulk}}+h_{x} X_{B}+\pi a h_{y} \mathbb{N},
$$

with the operators $\mathbb{N}$ and $\mathbb{S}_{ \pm}$in (22) satisfying the relations (24) and (25).

\subsection{Singularities of the hairpin boundary amplitude}

Looking at the right hairpin amplitude (17) as a function of complex variables $P$ and $Q$, one observes two sets of singularities. First one is a sequence of poles at $2 \mathrm{i} b P=0,-1,-2, \ldots$ due to the first gamma-factor in the numerator in (17). These poles admit straightforward interpretation in terms of potential divergences of the functional integral (7), with the non-compact boundary constraint (14), at the infinite end of the hairpin $X \rightarrow-\infty$ (see Ref. [1]). The second set is a string of poles at

$$
P_{k}=2 \mathrm{i} b k, \quad k=1,2,3 \ldots
$$

\footnotetext{
${ }^{7}$ It is important at this point that our definition of the hairpin (and of the paperclip) model involves uncompactified field $Y$. Also, the bound $\left|Y_{B}\right|<\pi a$ in (14) (and in (2) ) is essential. Without the bound, Eq.(14) (as well as Eq.(2) ) would define a series of disconnected curves, $Y \rightarrow Y+4 \pi a \mathbb{Z}$ copies of the original hairpin (or paperclip). Although the models of boundary interaction which involve more then one copy also deserve attention, they are different from the hairpin (paperclip) model as defined in [1], and we do not address them here.
} 
due to the second gamma-factor in (17). Note that in the weak-coupling limit of the hairpin model, i.e. at $b \rightarrow \infty$, these poles depart to infinity. Clearly, in terms of the hairpin functional integral these singularities represent nonperturbative effects. Instead, the poles (28) become most visible at small $b$, which is the weak coupling domain in the dual representation of the hairpin model, and indeed they admit simple interpretation in terms of the dual hairpin model. Since the boundary potential (21) vanishes in the limit $X \rightarrow-\infty$, there is a potential divergence of the functional integral associated with the dual hairpin ${ }^{8}$. Following [6], one can first integrate out the constant mode of the field $X$. This integration produces poles in $P$ exactly at the points (28) whose residues

$$
R_{k}=\mathrm{i} \operatorname{Res}_{P=2 \mathrm{i} b k}\left[Z_{\supset}(-\mathrm{i} P T,-\mathrm{i} Q T)\right] \quad(k=0,1,2 \ldots)
$$

are expressed through the integrals of the $2 k$-point correlation functions

$$
R_{k}=(2 \pi T)^{n k^{2}} \mathcal{T} \int \mathrm{d} \tau_{2 k} \cdots \mathrm{d} \tau_{1}\left\langle\left\langle\hat{V}_{\supset}\left(\tau_{2 k}\right) \cdots \hat{V}_{\supset}\left(\tau_{1}\right)\right\rangle\right\rangle_{0}
$$

where the $\tau$-ordering symbol $\mathcal{T}$ signifies that the integration is performed over the domain $1 / T \geq \tau_{2 k} \geq \cdots \geq \tau_{1} \geq 0$. In (30) $\hat{V}_{\supset}(\tau)=\mathrm{e}^{\tau \hat{H}_{0}} \hat{V}_{\supset} \mathrm{e}^{-\tau \hat{H}_{0}}$ is the unperturbed Matsubara operator associated with the boundary potential (22), and $\langle\langle\cdots\rangle\rangle_{0} \equiv \operatorname{Tr}\left[\cdots \mathrm{e}^{-\frac{\hat{H}_{0}}{T}}\right]$. Since the unperturbed Hamiltonian $\hat{H}_{0}$ involves no interaction between the boundary variables $\mathbb{S}_{ \pm}$and the fields $X, \tilde{Y}$, the expectation value in (30) factorizes in terms of these two parts of the system. In view of (22), it can be written in the form

$$
\begin{aligned}
R_{k}=\sum_{\epsilon_{i}= \pm} \operatorname{Tr}_{\rho}\left[\mathbb{S}_{\epsilon_{2 k}} \mathbb{S}_{\epsilon_{2 k-1}} \cdots \mathbb{S}_{\epsilon_{1}} \mathrm{e}^{\mathrm{i} \pi a Q \mathbb{N}}\right] \times \\
\mathcal{T} \int \mathrm{d} \tau_{2 k} \cdots \mathrm{d} \tau_{1}\left\langle\mathcal{V}_{B}^{\epsilon_{2 k}}\left(\tau_{2 k}\right) \cdots \mathcal{V}_{B}^{\epsilon_{1}}\left(\tau_{1}\right)\right\rangle_{0},
\end{aligned}
$$

which involves the traces over the space of states $\rho$ of the boundary degrees of freedom $\mathbb{S}_{ \pm}$, as well as the free-field expectation values $\langle\cdots\rangle_{0}$ of the boundary values $\mathcal{V}_{B}^{ \pm}(\tau)$ of the vertex operators (20). Thanks to the relations

\footnotetext{
${ }^{8}$ It is not difficult to write down the full functional integral for the dual hairpin model, which involves integration over the fields $X$ and $\tilde{Y}$, as well as the integral over the boundary spin $\mathbf{S}=\left(S_{+}, S_{-}, S_{3}\right)$ with the Wess-Zumino term (see, e.g., Ref. [5]). Such expression is not very useful for our analysis, except for the observation that the only terms in the full action which involve the constant mode of $X$ are the boundary potential term (21), and the term $\tilde{\mathscr{A}}_{\mathbf{h}}=\int_{0}^{1 / T} \mathrm{~d} \tau\left[h_{x} X_{B}(\tau)+\pi a h_{y} S_{3}(\tau)\right]$ responsible for the coupling to the external field $\left(h_{x}, h_{y}\right)$.
} 
(24), there are only two nonvanishing contributions to the sum in (31), with $\left(\epsilon_{1} \cdots \epsilon_{2 k}\right)=(+-+-\cdots+-)$ and $\left(\epsilon_{1} \cdots \epsilon_{2 k}\right)=(-+-+\cdots-+)$. With this observation, and using explicit form of the free-field correlators in (31), this expression can be brought to the form:

$$
\begin{aligned}
& R_{0}=\frac{\mathrm{g}_{D}^{2}}{2 \pi} \operatorname{Tr}_{\rho}\left[\mathrm{e}^{\mathrm{i} \pi a Q \mathbb{N}}\right], \\
& R_{k}=\frac{\mathrm{g}_{D}^{2}}{2 \pi}(2 \pi T)^{-k} F_{k} G_{k} \quad(k=1,2, \ldots),
\end{aligned}
$$

where

$$
F_{k}=\mathrm{e}^{\mathrm{i} \pi a Q} \operatorname{Tr}_{\rho}\left[\mathrm{e}^{\mathrm{i} \pi a Q \mathbb{N}}\left(\mathbb{S}_{-} \mathbb{S}_{+}\right)^{k}\right]=\mathrm{e}^{-\mathrm{i} \pi a Q} \operatorname{Tr}_{\rho}\left[\mathrm{e}^{\mathrm{i} \pi a Q \mathbb{N}}\left(\mathbb{S}_{+} \mathbb{S}_{-}\right)^{k}\right],
$$

and $G_{k}$ are given by the $2 k$-fold integrals

$$
\begin{aligned}
G_{k}= & \int_{0}^{2 \pi} \mathrm{d} u_{k} \int_{0}^{u_{k}} \mathrm{~d} v_{k} \int_{0}^{v_{k}} \mathrm{~d} u_{k-1} \cdots \int_{0}^{v_{2}} \mathrm{~d} u_{1} \int_{0}^{u_{1}} \mathrm{~d} v_{1} \times \\
& \prod_{j>i}^{k}\left[4 \sin \left(\frac{u_{j}-u_{i}}{2}\right) \sin \left(\frac{v_{j}-v_{i}}{2}\right)\right] \prod_{j \geq i}^{k}\left[2 \sin \left(\frac{u_{j}-v_{i}}{2}\right)\right]^{-4 b^{2}-1} \times \\
& \prod_{j>i}^{k}\left[2 \sin \left(\frac{v_{j}-u_{i}}{2}\right)\right]^{-4 b^{2}-1} 2 \cos \left[a Q\left(\pi+\sum_{i=1}^{k}\left(v_{i}-u_{i}\right)\right)\right] .
\end{aligned}
$$

The overall factor $\frac{\mathrm{g}_{D}^{2}}{2 \pi}$ in (32) appears because (31) involves unnormalized free-field correlation functions; here $\mathrm{g}_{D}=2^{-1 / 4}$ is well known "boundary entropy" factor [7] associated with the Dirichlet and von-Neumann boundary conditions for a free boson ${ }^{9}$.

Obviously, the way they are written above, the integrals (34) diverge for all positive $b^{2}$. As is common in conformal perturbation theory, we assume here a version of "analytic regularization", where the expressions are understood as analytic continuations of these integrals from the domain $\Re e b^{2}<0^{10}$. This procedure is performed in Appendix. Remarkably, the

\footnotetext{
${ }^{9}$ The $g$-factor of uncompactified boson $X$ with the von-Neumann boundary condition diverges. Formally, it involves the factor $\frac{\mathrm{g}_{\mathrm{D}}}{2 \pi} \mathrm{d} X_{0}$, where $X_{0}$ is the zero mode of $X$ [8]. In (30) the integration over $X_{0}$ is already performed - this integration was the origin of the poles (28). Additional factor $\mathrm{g}_{D}$ comes from the unperturbed partition function of $\tilde{Y}$. Since it is the T-duality transform of $Y$, its partition function equals to $\int \mathrm{d} \tilde{Q}\left\langle B_{N} \mid \tilde{Q}\right\rangle=\mathrm{g}_{D}$, where $\left\langle B_{N}\right|$ is the boundary state associated with the von Neumann boundary condition for the field $\tilde{Y}$.

${ }^{10}$ More generally, these divergences have to be canceled by adding a counterterm $M \mathrm{e}^{2 b X_{B}}$ (with the cutoff-dependent coefficient $M$ ) to the Hamiltonian (27).
} 
integrals (34) are evaluated in a closed form (the calculations are presented in Appendix),

$$
G_{k}=\frac{(2 \pi)^{k+1}\left(-4 b^{2}\right)^{-k} \Gamma\left(1-4 b^{2} k\right)}{k ! \Gamma\left(\frac{1}{2}-2 b^{2} k-a Q\right) \Gamma\left(\frac{1}{2}-2 b^{2} k+a Q\right)} \quad(k \geq 1) .
$$

Note that the $Q$-dependence of (35) is exactly as expected from (17), and moreover (32) coincide with the residues of (18), (17) at the points (28) provided

$$
\operatorname{Tr}_{\rho}\left[\mathrm{e}^{\mathrm{i} \pi a Q \mathbb{N}}\right]=2 \cos (\pi a Q)
$$

and

$$
\operatorname{Tr}_{\rho}\left[\left(\mathbb{S}_{-} \mathbb{S}_{+}\right)^{k}\right]=\operatorname{Tr}_{\rho}\left[\left(\mathbb{S}_{+} \mathbb{S}_{-}\right)^{k}\right]=\left(\frac{E_{*}}{2 \pi}\right)^{k} \quad(k=1,2 \ldots) .
$$

The equations (36) and (37) are sufficient to identify the representation $\rho$ of the algebra (24), (25) of the boundary degrees of freedom. From (36) one finds

$$
\operatorname{dim}(\rho)=2
$$

and

$$
\mathbb{N}^{2}=\mathbb{I}, \quad \operatorname{Tr}_{\rho}[\mathbb{N}]=0 .
$$

Next, the two-dimensional representation of the algebra must satisfy additional relation

$$
\left\{\mathbb{S}_{+}, \mathbb{S}_{-}\right\}=\frac{E_{*}}{2 \pi} \times \mathbb{I} .
$$

Indeed, $\rho$ is necessarily an irreducible representation of the algebra. As follows from Eqs.(24), (25), the anticommutator $\left\{\mathbb{S}_{+}, \mathbb{S}_{-}\right\}$commutes with $\mathbb{S}_{ \pm}$and $\mathbb{N}$, therefore it should be a constant in $\rho$. With Eq. (37) this implies the condition (40).

There is a unique (up to equivalence) two-dimensional representation of (24), (25), (40) which satisfies (39):

$$
\rho: \mathbb{S}_{+}=\sqrt{\frac{E_{*}}{2 \pi}} \sigma_{+}, \quad \mathbb{S}_{-}=\sqrt{\frac{E_{*}}{2 \pi}} \sigma_{-}, \quad \mathbb{N}=\sigma_{3},
$$

where $\sigma_{a}$ are conventional Pauli matrices. Thus we identify the boundary degree of freedom of the dual hairpin model with the spin $s=1 / 2$. Note that two eigenvalues of $\sigma_{3}$ are associated with two legs of the hairpin. Note also that according to (41) the operators $\mathbb{S}_{ \pm}$have dimension [energy $]^{\frac{1}{2}}$, as required by the balance of dimensions in Eq. (21) (in view of (3) the vertex operators $\mathrm{e}^{b X_{B} \pm \mathrm{i} a \tilde{Y}_{B}}$ have dimensions [energy $\left.]^{\frac{1}{2}}\right)$. 


\section{Dual to the paperclip model}

The dual representation for the paperclip model can be identified using similar line of arguments. The idea of the paperclip (2) being the composition of the left and right hairpins makes it natural to look for the Hamiltonian of the dual paperclip model in the form

$$
\hat{H}=\hat{H}_{\text {bulk }}+h_{x} X_{B}+\pi a h_{y} \mathbb{N}+\hat{V},
$$

where $\hat{H}_{\text {bulk }}$ is the same free-field bulk Hamiltonian (10), and

$$
\begin{aligned}
\hat{V}=\sqrt{\frac{E_{*}}{2 \pi}}\left[\mathbb{A}_{+} \mathrm{e}^{b X_{B}+\mathrm{i} a \tilde{Y}_{B}}+\mathbb{A}_{-} \mathrm{e}^{b X_{B}-\mathrm{i} a \tilde{Y}_{B}}+\right. \\
\left.\mathbb{B}_{+} \mathrm{e}^{-b X_{B}+\mathrm{i} a \tilde{Y}_{B}}+\mathbb{B}_{-} \mathrm{e}^{-b X_{B}-\mathrm{i} a \tilde{Y}_{B}}\right] .
\end{aligned}
$$

Here $\mathbb{A}_{ \pm}, \mathbb{B}_{ \pm}$and $\mathbb{N}$ are operators representing boundary degrees of freedom, which commute with the field operators $\mathbf{X}(\sigma), \boldsymbol{\Pi}(\sigma)$. Note that we have explicitly put the factor $\propto \sqrt{E_{*}}$ in (43), so that the operators $\mathbb{A}_{ \pm}, \mathbb{B}_{ \pm}$are dimensionless. Obviously, the first two terms in (43) are associated with the left hairpin component of the paperclip, i.e. the operators $\mathbb{A}_{ \pm}$play the

same role as $\mathbb{S}_{ \pm} / \sqrt{\frac{E_{*}}{2 \pi}}$ in (22). The last two terms in (43) are associated in a similar way with the dual form of the right hairpin. This correspondence suggests that the operators $\mathbb{B}_{ \pm}$, as well as $\mathbb{A}_{ \pm}$, satisfy the fermionic relations analogous to (24)

$$
\mathbb{A}_{ \pm}^{2}=0, \quad \mathbb{B}_{ \pm}^{2}=0
$$

In addition, they have to satisfy the commutation relations with $\mathbb{N}$

$$
\left[\mathbb{N}, \mathbb{A}_{ \pm}\right]= \pm 2 \mathbb{A}_{ \pm}, \quad\left[\mathbb{N}, \mathbb{B}_{ \pm}\right]= \pm 2 \mathbb{B}_{ \pm}
$$

and the anticommutation relations

$$
\left\{\mathbb{A}_{+}, \mathbb{A}_{-}\right\}=\left\{\mathbb{B}_{+}, \mathbb{B}_{-}\right\}=\mathbb{I} .
$$

Intuitively, these relations can be advocated by the same arguments that were considered in the previous section. The paperclip curve in Fig. 1 can be regarded as the combination of two nearly straight parallel D-branes connected to each other by the left and the right hairpin curves. Then, the presence of the vertex operators $\mathrm{e}^{b X_{B} \pm \mathrm{i} a \tilde{Y}_{B}}$ in (43) is the way how the dual representation reflects the possibility of passages from one straight brane to another via the connection at the right, i.e. at sufficiently large positive 
$X_{B}$. Likewise, the operators $\mathrm{e}^{-b X_{B} \pm \mathrm{i} a \tilde{Y}_{B}}$, which become significant at large negative $X_{B}$, describe the transitions between the nearly parallel branes via the connection at the left. This picture suggests that the space of states associated with the boundary degrees of freedom in (42), i.e. the supporting space of the representation $\rho$ of the above algebra, is two-dimensional, with two basic vectors (the eigenvectors of $\mathbb{N}$ ) corresponding to the two constituent straight branes. Then, Eq.(44) simply express the statement that there is s single copy of the paperclip curve (as is specified by the $Y_{B}$ bound in Eq.(2) $)$. Also, since $\rho$ is irreducible, and since in the limit $E_{*} \rightarrow 0$ we have to recover (40), the relations (46) follow.

It is easy to check that any two-dimensional representation $\rho$ of the algebra (44), (45) and (46) is equivalent to the following one:

$$
\rho: \quad \mathbb{A}_{ \pm}=\mathrm{e}^{ \pm \mathrm{i} \frac{\theta}{2}} \sigma_{ \pm}, \quad \mathbb{B}_{ \pm}=\mathrm{e}^{\mp \mathrm{i} \frac{\theta}{2}} \sigma_{ \pm},
$$

where $\theta$ is an arbitrary complex parameter. It is possible to show that this parameter must be real and, moreover, it coincides with the $\theta$-angle of the paperclip model.

The easiest way to verify this identification of $\theta$ is to analyze specific logarithmic divergences generated by the interaction (43). The divergences appear due to the singular term in the Operator Product Expansions

$$
\mathrm{e}^{b X_{B} \pm \mathrm{i} a \tilde{Y}_{B}}(\tau) \mathrm{e}^{-b X_{B} \mp \mathrm{i} a \tilde{Y}_{B}}\left(\tau^{\prime}\right)=\frac{1}{\left|\tau-\tau^{\prime}\right|}+\text { regular terms }
$$

and it is easy to check that in view of (47) they can be absorbed by local boundary counterterm

$$
-2 E_{*} \cos (\theta) \log \left(\Lambda / E_{*}\right) \quad \int \frac{\mathrm{d} \tau}{2 \pi},
$$

where $\Lambda$ is the UV cut-off. Exactly the same counterterm, with $\theta$ being the topological angle, is required in the original formulation of the paperclip model, where its role is to compensate for "small instanton" divergences (see [1] for details).

The above observation suggests that the instanton contributions of the original paperclip model are reproduced by certain terms of conformal perturbation theory of the dual model (42), (43). To be sure, the conformal perturbation theory, understood as a regular expansion in powers of $E_{*}$, can not be literally valid for this model. The model has many properties in common with the boundary sinh-Gordon model, and in view of the analysis in [9], one expects rather complicated structure of the UV expansion 
in (42). For example, in the case $h_{x}=0$ and at $T \gg E_{*}$ the partition function $\left.Z\left(h_{x}, h_{y} \mid T\right)\right|_{h_{x}=0}$ of the model (42) is expected to develop a large logarithmic term,

$$
Z\left(0, h_{y} \mid T\right)=G(Q \mid \kappa) \log \left(\frac{1}{\kappa}\right)+F(Q \mid \kappa),
$$

which derives from large $\left(\sim b^{-1} \log \left(\frac{1}{\kappa}\right)\right)$ fluctuations of the zero mode of the field $X$. The coefficient $G$ depends on $Q=\mathrm{i} \frac{h_{y}}{T}$ and

$$
\kappa=\frac{E_{*}}{2 \pi T} .
$$

Up to an overall factor $\kappa^{2 \kappa \cos (\theta)}$ (whose origin could be traced down to the singular term in Eq.(48)), it admits a small- $\kappa$ expansion in double series in powers of $\kappa$ and $\kappa^{\frac{1}{2 b^{2}}}$ (the term $F$ has a similar expansion). The term $\sim \kappa$ of this expansion is given by the integral

$$
\begin{aligned}
G(Q \mid \kappa)= & \frac{\mathrm{g}_{D}^{2}}{2 \pi b} \operatorname{Tr}_{\rho}\left[\mathrm{e}^{\mathrm{i} \pi a Q \mathbb{N}}\{1+\right. \\
& \left.\left.\mathcal{T} \int \mathrm{d} \tau_{2} \mathrm{~d} \tau_{1}\left\langle V_{\supset}\left(\tau_{2}\right) \hat{V}_{\subset}\left(\tau_{1}\right)+\hat{V}_{\subset}\left(\tau_{2}\right) \hat{V}_{\supset}\left(\tau_{1}\right)\right\rangle_{0}+\ldots\right\}\right],
\end{aligned}
$$

where $\hat{V}_{\supset}$ and $\hat{V}_{\subset}$ correspond to the first two terms and the second two terms on Eq.(43), respectively. Putting in explicit correlation functions of the exponential fields in (43) and evaluating the trace, one can bring this expression to a more explicit form

$$
G(Q \mid \kappa)=\frac{\mathrm{g}_{D}^{2}}{2 \pi b} 2 \cos (\pi a Q)(1+\kappa D(Q) \cos (\theta)+\ldots),
$$

where the factor $\cos (\theta)$ in the second term appears as the result of evaluation of the trace using Eqs.(47), and $D(Q)$ is the integral

$$
D(Q)=\frac{1}{2 \pi} \int_{0}^{2 \pi} \mathrm{d} u_{2} \int_{0}^{u_{2}} \mathrm{~d} u_{1} \frac{\cos \left(a Q\left(\pi-u_{2}+u_{1}\right)\right)}{\cos (\pi a Q) \sin \left(\frac{u_{2}-u_{1}}{2}\right)}+\text { counterterm }
$$

where $u_{1}$ and $u_{2}$ differ from $\tau_{1}$ and $\tau_{2}$ in (52) by a factor of $2 \pi T$. The integral logarithmically diverges when $u_{1} \rightarrow u_{2}$, but its divergent part cancels with the counterterm (49), and the $Q$-dependent finite part is evaluated explicitly, in terms of Euler's function $\psi(x)=\frac{\mathrm{d}}{\mathrm{d} x} \log \Gamma(x)$. As the result, the expansion of $G(Q \mid \kappa)$ has the form (53) with

$$
D(Q)=2 \log (\kappa)-\psi\left(\frac{1}{2}-a Q\right)-\psi\left(\frac{1}{2}+a Q\right) .
$$

With this, the coefficient in front of $\cos (\theta)$ in (53) exactly matches oneinstanton contribution to the paperclip partition function (see Eq.(110) of $[1])$. 


\section{Acknowledgments}

The authors are grateful to Vladimir V. Bazhanov, Vladimir A. Fateev and Alexei B. Zamolodchikov for discussions and interest to this work.

The research is supported in part by DOE grant \#DE-FG02-96 ER 40959. ABZ gratefully acknowledges kind hospitality of Laboratoire de Physique Théorique de l'Ecole Normale Supérieure, and generous support from Foundation de l'Ecole Normale Supérieure, via chaire Internationale de Recherche Blaise Pascal.

\section{Appendix: Calculation of the integral $G_{k}(\underline{34})$}

\subsection{Contour integral representation of $G_{k}$}

Up to overall factor, the integrand in (34) coincides with the free-field expectation value of product of $2 k$ chiral vertex operators

$$
V_{ \pm}=\mathrm{e}^{2 b X_{R} \pm 2 \mathrm{i} a Y_{R}}
$$

where $\mathbf{X}_{R}=\left(X_{R}, Y_{R}\right)$ in the exponent stands for the holomorphic part of the Bose field $\mathbf{X}(\sigma, \tau)=\mathbf{X}_{R}(\tau+\mathrm{i} \sigma)+\mathbf{X}_{L}(\tau-\mathrm{i} \sigma)$. As usual, the free-field expectation values are fully determined by the two-point functions,

$$
\left\langle X_{R}(\tau) X_{R}\left(\tau^{\prime}\right)\right\rangle_{0}=\left\langle Y_{R}(\tau) Y_{R}\left(\tau^{\prime}\right)\right\rangle_{0}=-\frac{1}{2} \log \left[\sin \left(\pi T\left(\tau-\tau^{\prime}\right)\right)\right] .
$$

It is useful to introduce the following set of integrated products

$$
J_{p}\left(\epsilon_{1} \cdots \epsilon_{p}\right)=\int_{\tau_{0}}^{\tau_{0}+1 / T} \mathrm{~d} \tau_{p} \int_{\tau_{0}}^{\tau_{p}} \mathrm{~d} \tau_{p-1} \cdots \int_{\tau_{0}}^{\tau_{2}} \mathrm{~d} \tau_{1} V_{\epsilon_{p}}\left(\tau_{p}\right) \cdots V_{\epsilon_{1}}\left(\tau_{1}\right)
$$

where $\left(\epsilon_{1} \cdots \epsilon_{p}\right)$ is a set of signs, $\tau_{0}$ is fixed real parameter, and integration is along the real axis. Clearly, the integral (34) is certain linear combinations of expectation values of $J_{2 k}\left(\epsilon_{1} \cdots \epsilon_{2 k}\right)$ with $\left(\epsilon_{1} \cdots \epsilon_{2 k}\right)$ being one of two alternating sign sequences $(+-+\cdots+-)$ and $(-+-\cdots-+)$.

As the first step in calculation we transform (58) into contour integrals. To do this we introduce the "screening operators", the integrals of the chiral vertex operators:

$$
x_{ \pm}=\frac{1}{q-q^{-1}} \int_{\tau_{0}}^{\tau_{0}+1 / T} \mathrm{~d} \tau \mathrm{e}^{2 b X_{R} \pm 2 \mathrm{i} a Y_{R}}
$$


where $^{11}$

$$
q=\mathrm{e}^{\mathrm{i} \pi n} .
$$

Here and bellow in this Appendix we use the notation from Ref. [1]:

$$
n \equiv 4 b^{2} .
$$

The following relations,

$$
\begin{aligned}
& \left(q-q^{-1}\right) x_{+} J_{2 k-1}(-\cdots-)=J_{2 k}(+\cdots-)-q^{k} J_{2 k}(-\cdots+) \\
& \left(q-q^{-1}\right) x_{-} J_{2 k-1}(-\cdots-)=0 \\
& \left(q-q^{-1}\right) x_{+} J_{2 k}(-\cdots+)=J_{2 k+1}(+\cdots+) \\
& \left(q-q^{-1}\right) x_{-} J_{2 k}(-\cdots+)=q^{k} J_{2 k+1}(-\cdots-)
\end{aligned}
$$

can be easily established by rearranging the integration domains. Additional set of relations is obtained from these by replacing $x_{ \pm} \rightarrow x_{\mp}$ and simultaneously changing all signs, $J_{p}\left(\epsilon_{1} \cdots \epsilon_{p}\right) \rightarrow J_{p}\left(-\epsilon_{1} \cdots-\epsilon_{p}\right)$. Recursively applying (62), one can prove that

$$
\begin{aligned}
& J_{2 k}(+\cdots-)=\left(q-q^{-1}\right)^{k} \frac{(-1)^{k} q^{-\frac{k(k+1)}{2}}}{[k]_{q} !}\left(q^{k}\left(x_{-} x_{+}\right)^{k}+\left(x_{+} x_{-}\right)^{k}\right) \\
& J_{2 k}(-\cdots+)=\left(q-q^{-1}\right)^{k} \frac{(-1)^{k} q^{-\frac{k(k+1)}{2}}}{[k]_{q} !}\left(q^{k}\left(x_{+} x_{-}\right)^{k}+\left(x_{-} x_{+}\right)^{k}\right),
\end{aligned}
$$

where the standard notations,

$$
[k]_{q} !=[1]_{q}[2]_{q} \ldots[k]_{q}, \quad[k]_{q}=\frac{q^{k}-q^{-k}}{q-q^{-1}},
$$

are used.

To make the next step more transparent we change to the new coordinate

$$
z=\mathrm{e}^{2 \pi \mathrm{i} T \tau} .
$$

The screening charges (59) become contour integrals in the variable $z$. More precisely, the action of the operators $x_{ \pm}$on any state created by a set of local insertions in the $z$-plane is written as the integral

$$
x_{ \pm}(\ldots)=\frac{1}{q-q^{-1}} \int_{C} d z V_{ \pm}(z)(\ldots),
$$

\footnotetext{
${ }^{11} \mathrm{As}$ it is known, the screening operators $x_{ \pm}$, together with the two zero mode operators, $\int_{\tau_{0}}^{\tau_{0}+1 / T} \mathrm{~d} \tau\left(a \partial_{\tau} X_{R} \pm \mathrm{i} b \partial_{\tau} Y_{R}\right)$, form the Chevelley basis of the Borel subalgebra of quantum superalgebra $U_{q, q}(\operatorname{sl}(2 \mid 1))[10]$.
} 
where the contour $C$ starts from the point $\zeta_{0}=\mathrm{e}^{2 \pi \mathrm{i} T \tau_{0}}$, goes around all the insertions in the counterclockwise direction, and then returns back to $\zeta_{0}$, as is shown in Fig.2. The integrand in (66) should be understood in terms of free-field operator product expansions. In general, the contour is not closed since operator product in the integrand is multivalued function of $z$. Using

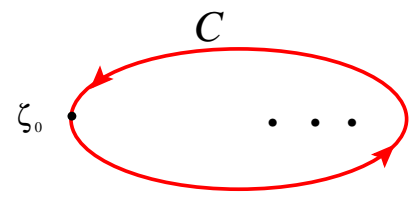

Figure 2: The integration contour in Eq. 666).

(63) the integral $G_{k}$ can be rewritten in the form:

$$
\begin{aligned}
& G_{k}=2(-1)^{k} q^{\frac{k^{2}}{2}} \frac{\left(q-q^{-1}\right)^{k}}{[k]_{q} !}\left[\cos \left(\pi\left(a Q+\frac{n k}{2}\right)\right) \times\right. \\
& \left.\left\langle\left(x_{+} x_{-}\right)^{k} V_{\lambda}(0)\right\rangle+\cos \left(\pi\left(a Q-\frac{n k}{2}\right)\right)\left\langle\left(x_{-} x_{+}\right)^{k} V_{\lambda}(0)\right\rangle\right],
\end{aligned}
$$

or more explicitly

$$
\begin{gathered}
G_{k}=\frac{2^{1-k} \mathrm{i}^{k} \mathrm{e}^{-\frac{\mathrm{i} \pi}{2} n k^{2}}}{\prod_{j=1}^{k} \sin (\pi n j)} \int_{C_{k}} \mathrm{~d} \zeta_{k} \int_{S_{k}} \mathrm{~d} z_{k} \ldots \int_{C_{1}} \mathrm{~d} \zeta_{1} \int_{S_{1}} \mathrm{~d} z_{1} \times \\
{\left[\cos \left(\pi\left(a Q+\frac{n k}{2}\right)\right)\left\langle V_{-}\left(\zeta_{k}\right) V_{+}\left(z_{k}\right) \ldots V_{-}\left(\zeta_{1}\right) V_{+}\left(z_{1}\right) V_{\lambda}(0)\right\rangle+\right.} \\
\left.\cos \left(\pi\left(a Q-\frac{n k}{2}\right)\right)\left\langle V_{+}\left(\zeta_{k}\right) V_{-}\left(z_{k}\right) \ldots V_{+}\left(\zeta_{1}\right) V_{-}\left(z_{1}\right) V_{\lambda}(0)\right\rangle\right],
\end{gathered}
$$

where the integration is over a set of contours $C_{j}, S_{j}(j=1,2, \ldots k)$ each starting at $\zeta_{0}$ and returning to the same point after going around the point 0 , and arranged so that $C_{k}$ lays entirely (except for the point $\zeta_{0}$ itself) inside $S_{k}, S_{k}$ lays inside $C_{k-1}, C_{k-1}$ lays inside $S_{k-1}$, etc., as is depicted in Fig. B. The expectation values in the integrand in (68) involves a certain vertex operator,

$$
V_{\lambda}=\exp \left(-\frac{n\left(\lambda_{+}+\lambda_{-}\right)}{2 b} X_{R}+\mathrm{i} \frac{n\left(\lambda_{-}-\lambda_{+}\right)}{2 a} Y_{R}\right)
$$




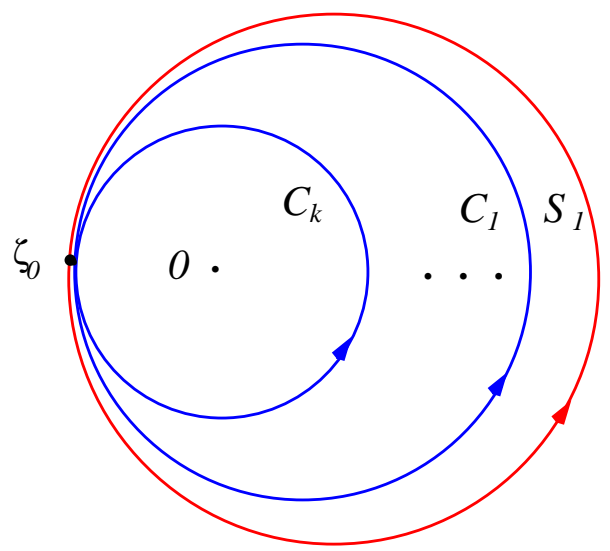

Figure 3: The integration contours in Eq.(68).

with $\lambda_{ \pm}$to be specified below, inserted at the origin $z=0$, so that

$$
\begin{aligned}
& \left\langle V_{-}\left(\zeta_{k}\right) V_{+}\left(z_{k}\right) \ldots V_{+}\left(z_{1}\right) V_{\lambda}(0)\right\rangle=\mathrm{i}^{-k} \mathrm{e}^{-\frac{\mathrm{i} \pi}{2} n k^{2}} \prod_{j=1}^{k} z_{j}^{n \lambda_{+}} \zeta_{j}^{n \lambda_{-}} \times \\
& \prod_{j>i}\left[\left(z_{j}-z_{i}\right)\left(\zeta_{i}-\zeta_{j}\right)\right] \prod_{j>i}\left[\left(\zeta_{j}-z_{i}\right)\left(z_{j}-\zeta_{i}\right)\right]^{-n-1} \prod_{j=1}^{k}\left(\zeta_{j}-z_{j}\right)^{-n-1} .
\end{aligned}
$$

We assume that the brunches of the power functions in (70) are chosen to give real positive values at

$$
0<z_{1}<\zeta_{1} \ldots<z_{k}<\zeta_{k} .
$$

In Eqs.(69), (170) the following notations are used

$$
n \lambda_{+}=\frac{k n-1}{2}+a Q+\varepsilon, \quad n \lambda_{-}=\frac{k n-1}{2}-a Q,
$$

where $\varepsilon$ is a complex parameter, which is assumed to be small, and eventually will be sent to zero.

\subsection{Combinatorics of the contour integrals}

Now let us introduce another set of integrated products of vertex operators,

$$
I_{p}\left(\epsilon_{1} \cdots \epsilon_{p}\right)=\int_{\zeta_{0}}^{0} \mathrm{~d} z_{p} \int_{\zeta_{0}}^{z_{p}} \mathrm{~d} z_{p-1} \cdots \int_{\zeta_{0}}^{z_{2}} \mathrm{~d} z_{1} V_{\epsilon_{1}}\left(z_{1}\right) \cdots V_{\epsilon_{p}}\left(z_{p}\right) V_{\lambda}(0)
$$


Here $V_{\lambda}$ is the vertex operator (69). Although the final result of the calculations below does not depend on a choice of the point $\zeta_{0}$, for convenience we choose it to be real and negative, and we assume that all the integrations in (73) are along the real axis. The operator products in (173) are multivalued functions of the integration variables, and we assume the same choice of the branch as in (70). Note that the integrals (73) are similar but different from (58), the main difference being in the form of integration contours.

The monodromies of the operator products in (73) are determined by symbolic relations

$$
\mathcal{A}_{C}\left[V_{ \pm}(z) V_{\lambda}(\zeta)\right]=q^{2 \lambda_{ \pm}} V_{ \pm}(z) V_{\lambda}(\zeta)
$$

and, in particular,

$$
\mathcal{A}_{C}\left[V_{+}(z) V_{-}(\zeta)\right]=q^{-2} V_{+}(z) V_{-}(\zeta)
$$

where the symbol $\mathcal{A}_{C}[\ldots]$ denotes analytic continuation in the variable $z$ along the contour $C$ shown on Fig. [4 Using these relations one can derive

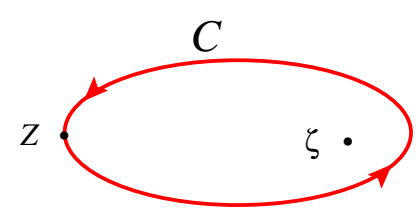

Figure 4: The contour of analytic continuation in Eq.(74).

the following identities:

$$
\begin{aligned}
x_{+} I_{2 k}(+\ldots-)= & -q^{\lambda_{+}-k}\left[\lambda_{+}\right]_{q} I_{2 k+1}(+\ldots+) \\
x_{+} I_{2 k}(-\ldots+)= & -q^{\lambda_{+}-k}\left[\lambda_{+}-k\right]_{q} I_{2 k+1}(+\ldots+) \\
x_{+} I_{2 k-1}(+\ldots+)= & 0 \\
x_{+} I_{2 k-1}(-\ldots-)= & -q^{\lambda_{+}-k}\left[\lambda_{+}-k\right]_{q} I_{2 k}(+\ldots-)- \\
& q^{\lambda_{+}-k}\left[\lambda_{+}\right]_{q} I_{2 k}(-\ldots+),
\end{aligned}
$$

as well as similar identities obtained from these by simultaneous change of the signs: $\left\{x_{ \pm} \rightarrow x_{\mp}, \lambda_{ \pm} \rightarrow \lambda_{\mp}, I_{p}\left(\epsilon_{1} \cdots \epsilon_{p}\right) \rightarrow I_{p}\left(-\epsilon_{1} \cdots-\epsilon_{p}\right)\right\}$. Here the action of the screening charges $x_{ \pm}$is defined by Eq. (66). The recursion (76) allows one to express the ordered integrals (73) in terms of the screening charges. In particular, one finds

$$
I_{2 k}(-\ldots+)=\frac{1}{c_{k}\left[\lambda_{+}\right]_{q}}\left(\left[\lambda_{+}-k\right]_{q}\left(x_{-} x_{+}\right)^{k}+\left[\lambda_{+}\right]_{q}\left(x_{+} x_{-}\right)^{k}\right) V_{\lambda}(\zeta),
$$


where

$$
c_{k}=(-1)^{k} q^{k\left(\lambda_{+}+\lambda_{-}-k\right)} \frac{[k]_{q} !\left[\lambda_{+}+\lambda_{-}-1\right]_{q} !}{\left[\lambda_{+}+\lambda_{-}-k-1\right]_{q} !} .
$$

Now assume that $\lambda_{ \pm}$are given by (72), and consider the limit $\varepsilon \rightarrow 0$. It is easy to check that at $\varepsilon=0$ the expression (73) becomes invariant with respect to simultaneous rescaling of all the integration variables, hence the integral develops logarithmic divergence at $z_{i} \rightarrow 0$. Thus, as the function of $\varepsilon, I_{2 k}(-+\cdots+)$ is expected to have a simple pole at $\varepsilon=0$. The appearance of the pole is very explicit in (77), since the coefficient $c_{k}$ vanishes at $\varepsilon=0$,

$$
c_{k} \rightarrow \varepsilon \frac{2 \pi \mathrm{i}}{q-q^{-1}}(-1)^{k}[k]_{q} ![k-1]_{q} ! .
$$

The representation (77) also makes it easy to isolate the residue at this pole,

$$
\begin{aligned}
& \lim _{\varepsilon \rightarrow 0} \varepsilon I_{2 k}(-\ldots+)=\frac{(-1)^{k}\left(q-q^{-1}\right)}{2 \pi \mathrm{i}[k]_{q} ![k-1]_{q} ! \cos \left(\pi\left(a Q+\frac{k n}{2}\right)\right)} \times \\
& \left(\operatorname { c o s } \left(\pi\left(a Q-\frac{k n}{2}\right)\left(x_{-} x_{+}\right)^{k}+\cos \left(\pi\left(a Q+\frac{k n}{2}\right)\left(x_{+} x_{-}\right)^{k}\right) V_{\lambda}(\zeta) .\right.\right.
\end{aligned}
$$

Comparing (80) with (67) we observe that the desired integrals $G_{k}$ can be expressed through such residues,

$$
\begin{aligned}
G_{k}= & 2^{k+1} \pi \mathrm{i}^{k} \mathrm{e}^{\frac{\mathrm{i} \pi}{2} n k^{2}} \prod_{j=1}^{k-1} \sin (\pi n j) \times \\
& \left.\cos \left(\pi\left(a Q+\frac{n k}{2}\right)\right) \lim _{\varepsilon \rightarrow 0} \varepsilon\left\langle I_{2 k}(-\ldots+)\right\rangle\right|_{\zeta=0} .
\end{aligned}
$$

Furthermore, it is easy to see that

$$
\begin{aligned}
& \left.\lim _{\varepsilon \rightarrow 0} \varepsilon\left\langle I_{2 k}(-\ldots+)\right\rangle\right|_{\zeta=0}=\frac{1}{k} \times \\
& \int_{0}^{1} \mathrm{~d} z_{2 k-1} \ldots \int_{0}^{z_{3}} \mathrm{~d} z_{2} \int_{0}^{z_{2}} \mathrm{~d} z_{1}\left\langle V_{-}(1) V_{+}\left(z_{2 k-1}\right) \ldots V_{+}\left(z_{1}\right) V_{\lambda}(0)\right\rangle
\end{aligned}
$$

and hence

$$
\begin{aligned}
& G_{k}=\frac{2^{k+1} \pi}{k} \prod_{j=1}^{k-1} \sin (\pi n j) \quad \cos \left(\pi\left(a Q+\frac{n k}{2}\right)\right) \times \\
& \int_{0}^{1} \mathrm{~d} z_{k} \int_{0}^{z_{k}} \mathrm{~d} \zeta_{k-1} \cdots \int_{0}^{\zeta_{1}} \mathrm{~d} z_{1} \prod_{j=1}^{k-1}\left[\zeta_{j}^{\frac{k n-1}{2}-a Q}\left(1-\zeta_{j}\right)\right] \prod_{j>i}\left(\zeta_{j}-\zeta_{i}\right) \times \\
& \prod_{j=1}^{k}\left[z_{j}^{\frac{k n-1}{2}+a Q}\left(1-z_{j}\right)^{-n-1}\right] \prod_{j>i}\left(z_{j}-z_{i}\right) \prod_{j, i}\left|z_{i}-\zeta_{j}\right|^{-n-1} .
\end{aligned}
$$




\subsection{Final step of the calculation}

Integrations over the variables $z_{1}, \ldots z_{k}$ in (83) can be eliminated by using the following identity ${ }^{12}$ :

$$
\begin{aligned}
& \int_{\zeta_{k-1}}^{\zeta_{k}} \mathrm{~d} z_{k} \int_{\zeta_{k-1}}^{\zeta_{k-2}} \mathrm{~d} z_{k-1} \cdots \int_{\zeta_{0}}^{\zeta_{1}} \mathrm{~d} z_{1} \prod_{j>i}\left(z_{j}-z_{i}\right) \times \\
& \prod_{j=0}^{k} \prod_{i=1}^{k}\left|\zeta_{j}-z_{i}\right|^{\alpha_{j}}=\frac{\prod_{j=0}^{k} \Gamma\left(1+\alpha_{j}\right)}{\Gamma\left(k+1+\sum_{j=0}^{k} \alpha_{j}\right)} \prod_{j>i}\left(\zeta_{j}-\zeta_{i}\right)^{\alpha_{i}+\alpha_{j}+1},
\end{aligned}
$$

where $\zeta_{k}>\zeta_{k-1}>\ldots>\zeta_{0}$ is an ordered set of real numbers. In the case under consideration $\zeta_{0}=0, \zeta_{k}=1$ and

$$
\alpha_{0}=\frac{k n-1}{2}+a Q, \quad \alpha_{j}=-n-1 \quad(j=1, \ldots k) .
$$

Thus

$$
G_{k}=\frac{2^{k+1} \pi^{2} D_{k-1}}{k \Gamma\left(\frac{1-k n}{2}-a Q\right) \Gamma\left(\frac{1-k n}{2}+a Q\right)} \Gamma^{k}(-n) \prod_{j=1}^{k-1} \sin (\pi n j),
$$

where $D_{k-1}$ is the Selberg integral $[12,13]$ :

$$
\begin{gathered}
D_{k-1}=\int_{0}^{1} \mathrm{~d} \zeta_{k-1} \int_{0}^{\zeta_{k-1}} \mathrm{~d} \zeta_{k-2} \ldots \int_{0}^{\zeta_{2}} \mathrm{~d} \zeta_{1} \prod_{j=1}^{k-1} \zeta_{j}^{(k-1) n-1}\left(1-\zeta_{j}\right)^{-2 n} \times \\
\prod_{j>i}\left(\zeta_{j}-\zeta_{i}\right)^{-2 n}=\frac{\pi^{k-1}(-n)^{-k} \Gamma(1-k n)}{(k-1) ! \Gamma^{k}(-n)} \frac{1}{\prod_{j=1}^{k-1} \sin (\pi n j)} \cdot
\end{gathered}
$$

Combining Eqs. 866) and (87) one arrives to (35).

\section{References}

[1] S.L. Lukyanov, E.S. Vitchev and A.B. Zamolodchikov, Nucl. Phys. B683, 423 (2004).

[2] S.L. Lukyanov, A.M. Tsvelik and A.B. Zamolodchikov, Nucl. Phys. B719, 103 (2005).

\footnotetext{
${ }^{12}$ This identity can be interpreted as the Dotsenko-Fateev representation [13] of the simple conformal block of Virasoro algebra with the central charge $c=-2$. A similar identity was used in Ref. [11].
} 
[3] V.A. Fateev, E. Onofri and Al.B. Zamolodchikov, Nucl. Phys. B406, 521 (1993).

[4] V.A. Fateev, Phys. Lett. B357, 397 (1995).

[5] A.M. Tsvelik, Quantum Field Theory in Condensed Matter Physics, Cambridge, UK: Univ. Press (1995).

[6] M. Goulian and M. Li, Phys. Rev. Lett. 66, 2051 (1991).

[7] I. Affleck and A. Ludwig, Phys. Rev. Lett. 67, 161 (1991).

[8] P. Fendley, H. Saleur and N.P. Warner, Nucl. Phys. B430, 577 (1994).

[9] Al.B. Zamolodchikov, unpublished notes (2001).

[10] R.B. Zhang, J. Phys. A: Math. Gen. 27, 817 (1994).

[11] P. Baseilhac and V. A. Fateev, Nucl. Phys. B532, 567 (1998).

[12] A. Selberg, Norsk. Mat. Tidsskr. 26, 71 (1944).

[13] V. Dotsenko and V. Fateev, Nucl. Phys. B251, 691 (1985). 